\title{
History independence of steady state in simultaneous two-phase flow through two-dimensional porous media
}

\author{
Marion Erpelding, ${ }^{1, *}$ Santanu Sinha, ${ }^{2}$ Ken Tore Tallakstad, ${ }^{1}$ Alex Hansen, ${ }^{2}$ Eirik Grude Flekkøy, ${ }^{1}$ and Knut Jørgen Måløy ${ }^{1}$ \\ ${ }^{1}$ Department of Physics, University of Oslo, P. O. Box 1048 Blindern, N-0316 Oslo, Norway \\ ${ }^{2}$ Department of Physics, Norwegian University of Science and Technology, N-7491 Trondheim, Norway
}

(Received 8 April 2013; published 7 November 2013)

\begin{abstract}
It is well known that the transient behavior during drainage or imbibition in multiphase flow in porous media strongly depends on the history and initial condition of the system. However, when the steady-state regime is reached and both drainage and imbibition take place at the pore level, the influence of the evolution history and initial preparation is an open question. Here, we present an extensive experimental and numerical work investigating the history dependence of simultaneous steady-state two-phase flow through porous media. Our experimental system consists of a Hele-Shaw cell filled with glass beads which we model numerically by a network of disordered pores transporting two immiscible fluids. From measurements of global pressure evolution, histograms of saturation, and cluster-size distributions, we find that when both phases are flowing through the porous medium, the steady state does not depend on the initial preparation of the system or on the way it has been reached.
\end{abstract}

DOI: 10.1103/PhysRevE.88.053004

PACS number(s): 47.56.+r, 47.55.dd, 47.55.Ca, 89.75.Fb

\section{INTRODUCTION}

Understanding the physical mechanisms underlying multiphase flow in porous media is crucial to a wide variety of industrial and environmental problems, such as oil recovery, $\mathrm{CO}_{2}$ transport and storage, or groundwater management. At the macroscopic scale, the flow of immiscible fluids in a porous material is described by specifying the relations between global quantities such as flow rate, pressure gradient, or fluid saturation. At the pore scale, this flow is governed by the competition between capillary, viscous, and gravitational forces. Understanding the link between the two levels of description requires relating the position and shape of the interface(s) between the two phases to the values of the macroscopic variables. From an experimental point of view, two-dimensional model porous media providing direct porescale visualization of the flow structures are ideal tools to study multiphase flow mechanisms and their relations with global quantities in controlled, laboratory-scale situations. Over the past decades, microchannel networks etched in transparent plates [1] or prepared using molding techniques [2] and porous Hele-Shaw cells consisting of a layer of beads between two parallel plates [3] have become classical tools, to which improvements have been constantly proposed $[4,5]$. Experimental observations have been explained through extensive numerical simulations based on network models [6-10] and lattice Boltzmann methods [11-17], statistical models [18-20], and differential equations [21]. Most of the research in this area has been focused upon transient phenomena, i.e., drainage or imbibition-arising when one phase displaces the other in a porous medium. The relation

*marion.erpelding@fys.uio.no

Published by the American Physical Society under the terms of the Creative Commons Attribution 3.0 License. Further distribution of this work must maintain attribution to the author(s) and the published article's title, journal citation, and DOI. between macroscopic flow parameters, fluid morphology, and the stability of the interface between the two phases has been thoroughly observed [3,4] and successfully modeled [18,19].

In this article, we deal with steady-state flow, where many questions are yet to be answered. In the steady-state regime, two phases are injected simultaneously into the porous medium and one observes that one or both are fragmented and transported in the form of clusters of various sizes, forming a complex flow pattern with multiple interfaces. After a characteristic time, the system reaches a steady state in which the macroscopic flow variables fluctuate around constant values. The usual distinction between drainage and imbibition is irrelevant to describe steady-state two-phase flow, in which both processes occur simultaneously. New approaches are thus needed to understand this regime. In an effort to bring new insight, experimental and numerical studies have investigated the relations between macroscopic flow variables, and different models have been proposed to relate them to pore-scale flow mechanisms: Payatakes and co-workers carried out detailed experimental, numerical, and theoretical studies of steady-state two-phase flow, with emphasis on the determination of relative permeabilities $[1,9,10,22-25]$. The steady-state characteristics of macroscopic flow properties have also been investigated numerically by Knudsen and Hansen [26]. A power-law relation between pressure and steady-state flow rate has been observed experimentally by Tallakstad et al. in a two-dimensional (2D) system [27,28], and by Rassi et al. in a 3D system [29]. Very recently, the relation between the steady-state flow rate and pressure drop has been derived analytically for two-phase flow through single capillaries [30] and through porous media [27,28,31] and also supported by extensive numerical simulation. Distributions of nonwetting or wetting clusters in the steady state have also been studied experimentally by Tallakstad et al. [27,28] and numerically by Ramstad and Hansen [32], and critical exponents were measured. It is worth noting, though, that the comparison of experimental and numerical results is not always straightforward, due to the different boundary conditions used in the two cases. Yet from the point of 
view of statistical physics, the existence of a genuine steady state is very promising for building a thermodynamiclike theoretical description of the system. In that context, it is crucial to determine whether the steady state is independent of the history of the process, or in other words, whether it is a real state in a thermodynamic sense [33]. It is well known that when drainage and imbibition occur successively, the relative permeabilities become history dependent and the pressure-saturation curves display a hysteresis [34,35], the underlying pore-scale mechanisms of which are known. In addition to the well-known magnetic and elastic systems, such history dependence and hysteresis has also been observed in different flow processes like hydrodynamic heat flow [36] and particle flow through random media [37]. However, in the case of two-phase flow through porous media, it is not trivial to predict whether such a hysteresis will come into play in the steady-state situation when drainage and imbibition occur simultaneously. It was proposed that a thermodynamiclike description for simultaneous two-phase flow in porous media can be sketched [33] if the flow was history independent.

Here, we present an extensive experimental and numerical study in order to investigate the history independence of the steady state in a $2 \mathrm{D}$ configuration. Our experimental system consists of a porous Hele-Shaw cell of relatively high porosity in which we simultaneously inject air and a viscous water-glycerol solution, thus exploring the regime of high viscosity contrast. We then compare steady states obtained for a given flow rate with different initial conditions. We model the system by a network of disordered pores transporting two immiscible fluids. From pressure measurements and analysis of the statistical properties of the flow patterns, we observe no history dependence for the steady state.

\section{EXPERIMENTAL SETUP}

We use a two-dimensional, transparent, porous Hele-Shaw cell. This experimental setup, shown on Figs. 1 and 2, has been described in detail in [27] and we recall its main features here. The porous medium consists of a random monolayer of glass beads, $1 \mathrm{~mm}$ in diameter, spread between the sticky sides of two sheets of contact paper. Its lateral boundaries are sealed with silicon glue. Attached on top of this layer, a Plexiglas plate with etched flow channels allows injection and evacuation of fluids into and from the porous matrix. A pressure cushion (see [27] for details) placed below the porous medium and a thick glass plate on top prevent the system from bending when the pressure increases as fluids are injected. Clamps placed all around the setup maintain all the layers together. This way, we obtain a porous medium of constant thickness $a=1 \mathrm{~mm}$, length $L=85 \mathrm{~cm}$, and width $W=42 \mathrm{~cm}$ in which the beads remain immobile. The porosity $\phi$ and absolute permeability $\kappa_{0}$ of the medium are found experimentally to be $\phi=0.63$ and $\kappa_{0}=1.95 \times 10^{-5} \mathrm{~cm}^{2}$ [27].

We use the same fluid pair as Tallakstad et al. [27], namely, air as the nonwetting phase and a viscous water-glycerol solution (15-85\% in mass) as the wetting phase. The latter is dyed in black with $0.1 \%$ Negrosine to obtain a good contrast on the experimental images (see Fig. 3). As pointed out by Tallakstad et al., the use of air as one of the phases introduces more complexity in the two-phase flow problem

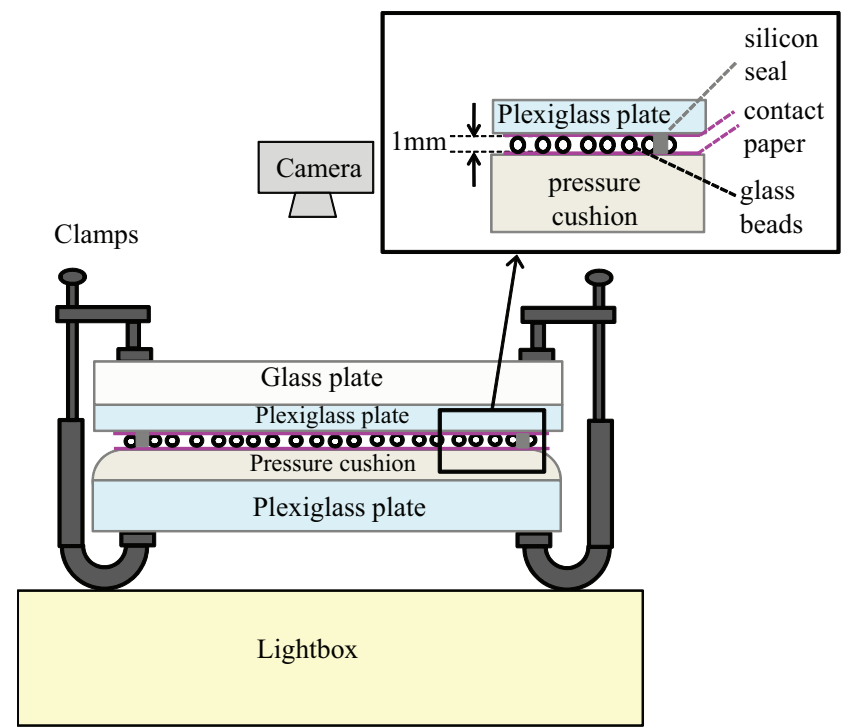

FIG. 1. (Color online) Sketch of the experimental setup: The 2D porous matrix consists of a disordered monolayer of glass beads spread between two sheets of contact paper. The boundaries are sealed with silicon glue. The upper part of the system consists of a Plexiglas plate with drilled inlet and outlet flow channels. A pressure cushion and a thick glass plate placed below and above the porous matrix ensure the overall rigidity of the system and maintain its thickness constant. Clamps maintain all the layers together. A lightbox illuminates the system from below and a digital camera is placed above to record images of the flow structure.

for two reasons: First, it yields a high viscosity contrast with the glycerol solution, and second, its compressibility gives rise to rapid bursts or avalanches [27]. However, from an experimental point of view, it has the huge advantage of allowing us to reuse the same porous model for all experiments. Indeed, it can easily be flushed out, making it possible to obtain reproducible initial conditions. Therefore, we find it fully convenient for the present study. As shown on Fig. 2, the two phases are injected simultaneously, using the same syringe pump, from 15 syringes (seven of air and eight of water-glycerol solution), each connected to one of the 15 inlet nodes of the porous model. We have checked experimentally that the results in terms of history dependence of the steady state are not modified if the two fluids are mixed in a $\mathrm{T}$ junction before entering the model. In the following, we denote by $Q$ the total flow rate, while $Q_{\mathrm{w}}=(8 / 15) Q$ and $Q_{\text {nw }}=(7 / 15) Q$ denote the wetting and nonwetting flow rates, respectively. To account for small temperature variations due to the heat released by the lightbox (see Fig. 1), we monitor the temperature of the wetting phase at the outlet of the model. The viscosity $\mu_{\mathrm{w}}$ of the wetting phase is deduced accordingly using the empirical formula given in [38]. In the series of experiments presented here, the measured temperatures are in the range $24.4-29.3{ }^{\circ} \mathrm{C}$, giving $0.083>\mu_{\mathrm{w}}>0.062 \mathrm{Pas}$. The viscosity of air being $\mu_{\mathrm{nw}} \approx 1.9 \times 10^{-5} \mathrm{~Pa}$ s, the viscosity ratio $M=\mu_{\mathrm{nw}} / \mu_{\mathrm{w}}$ of the order of $10^{-4}$ in all experiments.

With this setup, the total flow rate $Q$ and the fractional flow $F_{\mathrm{w}}=Q_{\mathrm{w}} / Q$ are controlled flow variables. The volumes of wetting and nonwetting fluids present in the porous matrix, $V_{\mathrm{w}}$ and $V_{\mathrm{nw}}$, are free to vary with time. Thus, the saturations 


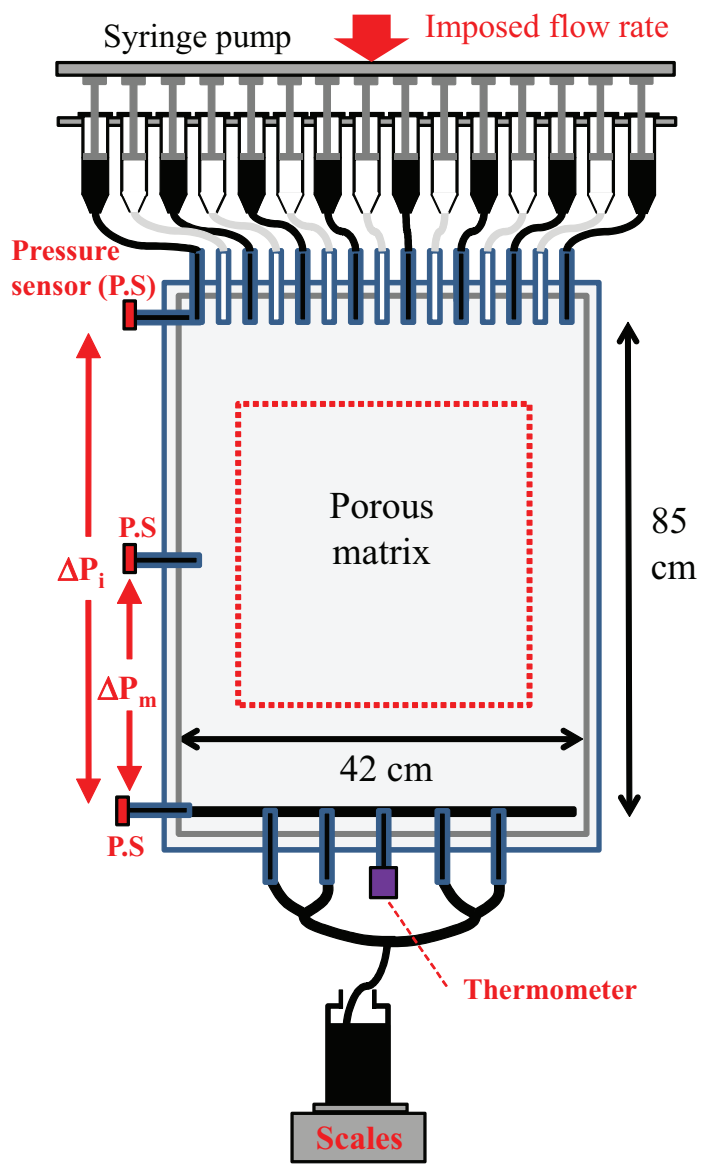

FIG. 2. (Color online) Sketch of the experimental setup with the injection system. The two phases are contained in 15 syringes, each connected to one of the 15 inlet nodes of the porous model (seven syringes of air represented in white and eight syringes of waterglycerol solution in black). The same syringe pump is used to inject both phases simultaneously. The dotted lines give the dimensions of the area studied by image analysis (note that proportions are not respected).

$S_{\mathrm{w}}=V_{\mathrm{w}} / V$ and $S_{\mathrm{nw}}=V_{\mathrm{nw}} / V$, where $V$ is the total pore volume, are free to fluctuate. The flow is characterized by the capillary number

$$
\mathrm{Ca}=\frac{\mu_{\mathrm{w}} Q_{\mathrm{w}}}{\gamma A},
$$

where $\mu_{\mathrm{w}}$ is the wetting phase viscosity, $Q_{\mathrm{w}}$ is the total wetting fluid flow rate, $\gamma \approx 6.4 \times 10^{-2} \mathrm{~N} / \mathrm{m}$ is the interfacial tension between the two phases [27], and $A=W a \phi$ is the cross section of the porous matrix. In the present experiments, we have explored the range $3.33 \times 10^{-6} \leqslant \mathrm{Ca} \leqslant 1.13 \times 10^{-4}$. The highest experimental value of $\mathrm{Ca}$ is set by the maximum pressure that the porous model can hold. However, as we will see in Sec. IV, we have also explored higher values of $\mathrm{Ca}$ in numerical simulations, namely, $1.92 \times 10^{-5} \leqslant \mathrm{Ca} \leqslant$ $7.0 \times 10^{-2}$. In this range, we always measure a significant pressure drop in the wetting phase, showing that we are in a flow regime in which viscous effects cannot be neglected.

Our analysis of steady-state flow and its history dependence relies on two kinds of information: measurements of the pressure inside the model, and pictures of the flow pattern. We

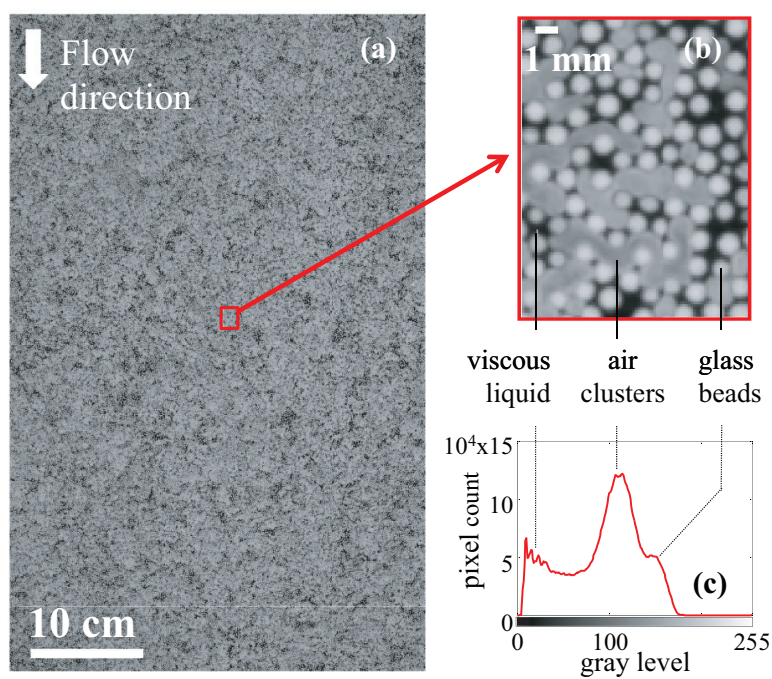

FIG. 3. (Color online) (a) Example of steady-state image $(2400 \times$ 3800 pixels): The flow pattern is made of air clusters (gray) of various sizes surrounded by the viscous liquid (black). (b) Zoom: The high image resolution makes it possible to distinguish glass beads (bright gray), air clusters (gray), and viscous liquid (black). These three phases yield three "peaks" on the grayscale image histograms, as illustrated by (c). The height of the peaks contains information about the saturation of the system.

measure the pressure $P(t)$ in the wetting phase as a function of time $t$ using flow-through pressure sensors (SensorTechnics 26PC0100G6G) placed at three different points of the model as indicated on Fig. 2. The porous model is lit from below using a lightbox, and images of the flow structure are recorded regularly using a Nikon D200 digital reflex camera giving $2592 \times 3872$ pixel images with a spatial resolution of $8 \times$ 8 pixels $/ \mathrm{mm}^{2}$. Before further processing, images are cropped to remove boundaries, leaving us with $2400 \times 3800$ pixels on the final images. Figure 3(a) shows an example of a steady-state image. The area of the imaged zone (see Fig. 2) is large enough to contain many air clusters of various sizes. As illustrated by Fig. 3(b), the high image resolution makes it possible to distinguish glass beads, air clusters, and viscous liquid. Each of these phases gives rise to a peak on the grayscale image histogram [see Fig. 3(c)]. The heights of these peaks contain information about the proportions of wetting and nonwetting fluids in the system, and thus about the saturations $S_{\mathrm{w}}$ and $S_{\mathrm{nw}}$. In all experiments, the smallest air clusters observed have sizes comparable with the pore size.

\section{EXPERIMENTAL RESULTS AND DISCUSSION}

\section{A. Principle of the experiments}

We investigate the history dependence of steady-state flow by comparing steady states obtained at the same flow rate but with different initial conditions. For this, we have performed experiments in which the flow rate is modified twice, as illustrated by Fig. 4(a). The porous model is initially filled with the wetting phase only. Then both phases are injected simultaneously at a fixed flow rate $Q_{1}$. Once the system has reached a steady state $\left(\mathrm{ss}_{1}\right)$, we abruptly change the flow rate to a different value $Q_{2}$ and wait until a new steady state is 

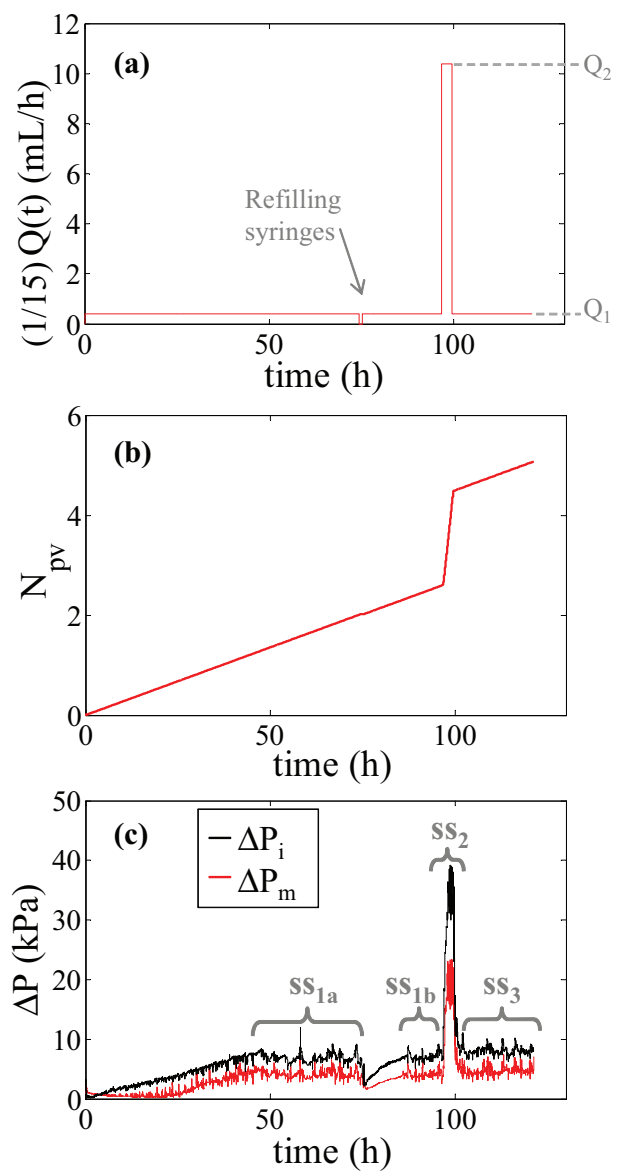

FIG. 4. (Color online) Temporal variation of imposed and measured experimental variables for a typical experiment (Expt. 3; see Table I): (a) Temporal variation of the imposed flow rate (1/15) $Q$ for one syringe. (b) Ratio of the total injected fluid volume to the available pore volume, computed from the imposed flow rate using Eq. (2). (c) Corresponding pressure drop measurements $\Delta P_{i}$ [black (upper) line] and $\Delta P_{m}$ [red (lower) line]. Plateaus characterize the different steady states (ss). The sudden drop around $t=75 \mathrm{~h}$ is an experimental artifact due to the refilling of the syringes, as explained in the text. Steady states obtained before and after refilling are distinguished using the subscripts $a$ and $b$.

established $\left(\mathrm{ss}_{2}\right)$. Finally, we change the flow rate back to its initial value $Q_{1}$ and let the system evolve towards a third steady state $\left(\mathrm{ss}_{3}\right)$. We have used different pairs $\left\{Q_{1}, Q_{2}\right\}$, listed in Table I, to obtain different magnitudes and signs of $\Delta Q=Q_{1}-Q_{2}$. We compare $\mathrm{ss}_{1}$ and $\mathrm{ss}_{3}$ using three criteria: The average pressure drop across the system, the fluid saturations, and the size distribution of the air clusters.

From the pressures measured at the inlet, middle, and outlet of the system, we compute the pressure drops $\Delta P_{i}(t)=$ $P_{\text {inlet }}(t)-P_{\text {outlet }}(t)$ and $\Delta P_{m}(t)=P_{\text {middle }}(t)-P_{\text {outlet }}(t)$ (see Fig. 2). Figure 4(c) shows a plot of these quantities for a typical experiment. After a transient characterized by a linear increase, both pressure drops stabilize and fluctuate around constant average values. This behavior is identical to what has been observed previously [27] and defines steady state $1\left(\mathrm{ss}_{1}\right)$. The fluctuations of $\Delta P$ in steady state reflect the dynamics of the nonwetting clusters-transport, mergings, and snapoffs-as
TABLE I. Experimental parameters and corresponding measurements. $Q_{1}$ and $Q_{2}$ refer to the total imposed flow rates (i.e., for 15 syringes). $\chi_{\text {inlet }}$ and $\chi_{\text {outlet }}$ are computed from the measured pressure drops according to Eq. (3). Note that experiments Expt. 1 and Expt. 6 are performed using the same parameters, illustrating the reproducibility of the results.

\begin{tabular}{lcccc}
\hline \hline Experiment & $Q_{1}(\mathrm{ml} / \mathrm{h})$ & $Q_{2}(\mathrm{ml} / \mathrm{h})$ & $\chi_{\text {inlet }}$ & $\chi_{\text {middle }}$ \\
\hline Expt. 1 & 6.15 & 61.1 & 0.91 & 0.31 \\
Expt. 2 & 61.1 & 6.15 & 0.96 & 0.93 \\
Expt. 3 & 6.15 & 156 & 1.89 & 1.12 \\
Expt. 4 & 156 & 6.15 & 2.86 & 2.31 \\
Expt. 5 & 15.0 & 30.5 & 0.67 & 0.04 \\
Expt. 6 & 6.15 & 61.1 & 0.19 & 0.04 \\
\hline \hline
\end{tabular}

the system explores different configurations [27,28]. Image analysis shows that this steady-state dynamics preserves both the average saturation and the average size distribution of the clusters (see Sec. III B). Immediately after we change the flow rate to the value $Q_{2}>Q_{1}$, both pressure drops display a very rapid increase, and a new steady state $\left(\mathrm{ss}_{2}\right)$, characterized by higher values of $\Delta P$, is quickly established. When we change the flow rate back to $Q_{1}$, we observe again a rapid variation of the pressure drops towards a third plateau defining steady state $3\left(\mathrm{ss}_{3}\right)$.

Because experiments are performed at slow flow rates, and to obtain enough statistics on the measurements, they must typically run for several days. Indeed, the quality of the statistics is determined by the ratio $N_{\mathrm{pv}}(t)$ of the total volume of fluids injected into the system to the total pore volume of the porous matrix, namely,

$$
N_{\mathrm{pv}}(t)=\frac{Q(t) t}{W L a \phi},
$$

where $Q$ denotes the total flow rate, $t$ denotes time, and $W, L$, $a$, and $\phi$ are the width, length, thickness, and porosity of the porous matrix, respectively. Figure 4 (b) shows $N_{\mathrm{pv}}$, calculated from the imposed flow rates as a function of time, for a typical experiment. In all the experiments presented here, the durations of the steady states correspond to the injection of 0.3 to 1.4 pore volumes into the system. As a consequence, it is necessary to refill the syringes one or several times in the course of an experiment. This is systematically performed using the following protocol: The outlet and inlets of the model are closed and the syringe pump is stopped immediately. The refilling process takes approximately $20 \mathrm{~min}$. Because the vents used to close the model are not perfectly airproof, the pressure in the system relaxes towards atmospheric pressure during this process, explaining the sudden drop of $\Delta P$ observed on Fig. 4(c). However, by looking at the pictures recorded throughout the process, we have checked that this does not affect the flow structure, which remains immobile over the duration of the refilling procedure. Furthermore, we observe that once the syringe pump is restarted, $\Delta P$ retrieves its initial value after a delay originating from the compressibility of air. Thus this refilling procedure does not affect the results of the experiments. In the following, when necessary, we 

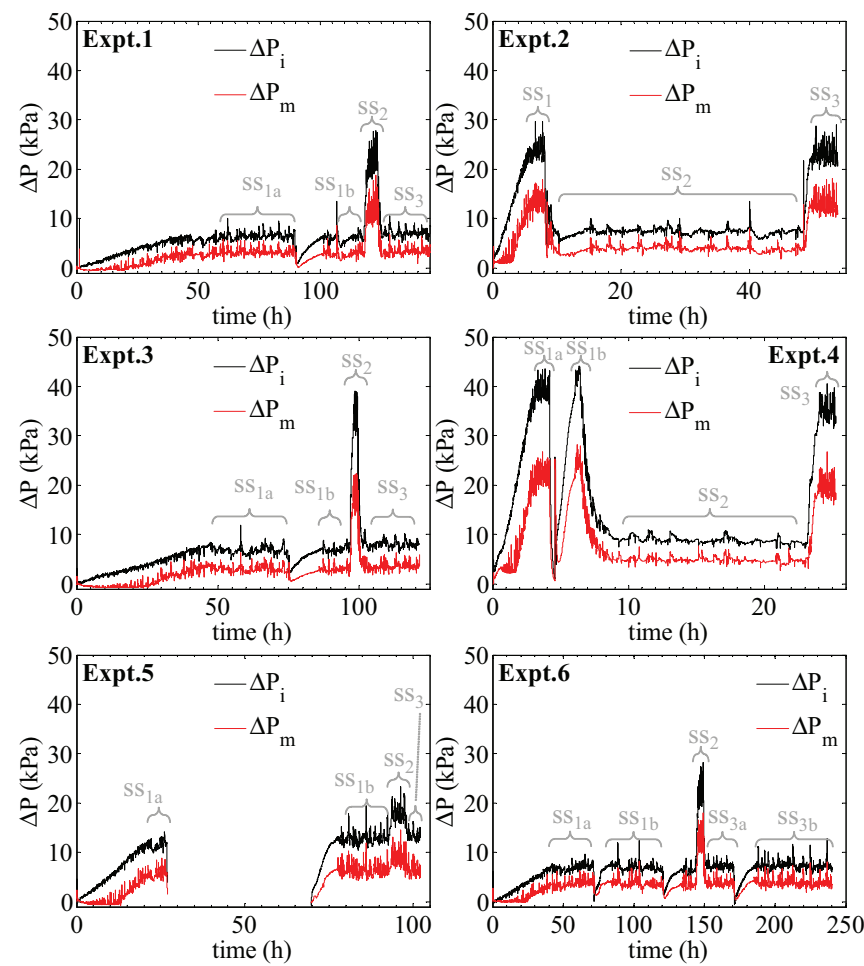

FIG. 5. (Color online) Measured pressure drops $\Delta P_{i}$ [black (upper) line $]$ and $\Delta P_{m}$ [red (lower) line] for the six experiments listed in Table I. The labels $\mathrm{ss}_{1}$, etc. on the plots indicate the different steady states. Note that because these various steady states are obtained with different flow rates, their lengths are different in time. However, the duration of each steady state always corresponds to the injection of 0.3 to 1.4 times the pore volume of the system. The lack of data between the 25th and 75th hours for Expt. 5 corresponds to the interruption of the experiment for pure convenience reasons. We do not expect this interruption to affect the results, based on the comparison between $\mathrm{ss}_{1 b}$ and $\mathrm{ss}_{3}$.

distinguish steady states obtained before and after refilling using the subscripts $a$ and $b$, respectively [see Fig. 4(c)].

\section{B. History independence of pressure drops}

Figure 5 shows the temporal evolution of the pressure drops $\Delta P_{i}$ and $\Delta P_{m}$ for the six experiments listed in Table I. In all cases, we observe a behavior similar to what has been described in the previous section, namely, rapid variations of $\Delta P$ upon changes of flow rate between plateaus characterizing steady states. Most importantly, we observe that whatever the value and sign of $\Delta Q$, the pressure drops are similar in $\mathrm{ss}_{1}$ and $\mathrm{ss}_{3}$. This suggests that the steady-state pressure drop $\Delta P_{\mathrm{ss}}$ depends only on the imposed flow rate and not on the history of the system. Note also that for a given flow rate, $\Delta P_{\mathrm{ss}}$ values are reproducible from one experiment to the next, regardless of how steady state has been reached.

To obtain a quantitative indication of this history independence, we compute average steady-state pressure drop values $\left\langle\Delta P_{\mathrm{ss}}\right\rangle$ over the duration of each steady state; thus over periods of time corresponding to the injection of 0.3 to 1.4 pore volumes in the system, as already indicated earlier. For each experiment, we compare the variation of $\left\langle\Delta P_{\mathrm{ss}}\right\rangle$ between $\mathrm{ss}_{1}$ and $\mathrm{ss}_{3}$ to the fluctuations of $\Delta P_{\mathrm{ss}}$ within these two states. For this we compute the ratio

$$
\chi=\left|\frac{\left\langle\Delta P_{\mathrm{ss}_{1}}\right\rangle-\left\langle\Delta P_{\mathrm{ss} 3}\right\rangle}{0.5\left[\sigma\left(\Delta P_{\mathrm{ss}_{1}}\right)+\sigma\left(\Delta P_{\mathrm{ss}_{3}}\right)\right]}\right|,
$$

where $\langle\cdots\rangle$ represents a temporal average over the duration of a steady state and $\sigma\left(\Delta P_{\mathrm{ss}_{1}}\right) \approx \sigma\left(\Delta P_{\mathrm{ss}_{3}}\right)$ are the standard deviations of $\Delta P_{\mathrm{ss}}$ in $\mathrm{ss}_{1}$ and $\mathrm{ss}_{3}$. The values of $\chi$ computed from the inlet and middle pressure drops for the different experiments are reported in Table I. As can be observed, almost all of these values are smaller than 1 . Slightly higher values are observed in Expt. 3 and Expt. 4. However, our experimental temperature data suggest that temperature-induced viscosity fluctuations most likely explain this fact. Indeed, the largest temperature variations both between $\mathrm{ss}_{1}$ and $\mathrm{ss}_{3}$ and within $\mathrm{ss}_{1}$ or $\mathrm{ss}_{3}$ occur for these two experiments. Therefore, we find that within the precision of our measurements, the steady-state pressure drop values are history independent.

\section{History independence of saturation and nonwetting cluster-size distributions}

Pressure measurements suggest that the observed steady state depends only on the imposed flow rate. However, these measurements do not give us detailed spatial information. Thus, we now analyze the images of steady-state flow patterns. As explained earlier (see Fig. 3), the flow structure consists of clusters of the nonwetting phase, air, surrounded by the wetting viscous water-glycerol solution. As air clusters are transported through the porous medium, they are fragmented or merged, giving many different realizations of the flow pattern. However, within a steady state, the saturation and the size distribution of air clusters remain constant on average [27]. Thus, as we did for pressure drops, we compute average steadystate saturations and cluster-size distributions and compare their properties in $\mathrm{ss}_{1}$ and $\mathrm{ss}_{3}$. To obtain good statistics on these quantities, we have chosen the frame rates so that successive images sample different realizations of the flow pattern. All averages are computed over series of images, typically 100 , spanning a time range corresponding to the injection of $1 / 5$ of the pore volume at the minimum.

As explained in Sec. II, the grayscale histograms of the raw images give us a direct indication of the proportions of the two phases in the system, and thus of saturation (see Fig. 3). Figure 6 shows average steady-state histograms for the six experiments listed in Table I. It is clear on all these graphs that the histograms corresponding to $\mathrm{ss}_{1}$ and $\mathrm{ss}_{3}$ can be distinguished from those corresponding to $\mathrm{ss}_{2}$ : Indeed, as expected, the saturation depends on the flow rate, as reflected by different air and liquid "peak" heights on the histograms. However, histograms are similar in $\mathrm{ss}_{1}$ and $\mathrm{ss}_{3}$, suggesting that the steady-state saturation is also history independent. It is important to note that histograms are directly obtained from raw images without prior processing, which excludes eventual artifacts due to image processing. However, they do not give us any information about the spatial repartition of the two phases in the system.

To obtain this information, we process the images to identify air clusters and compute their size distributions. 

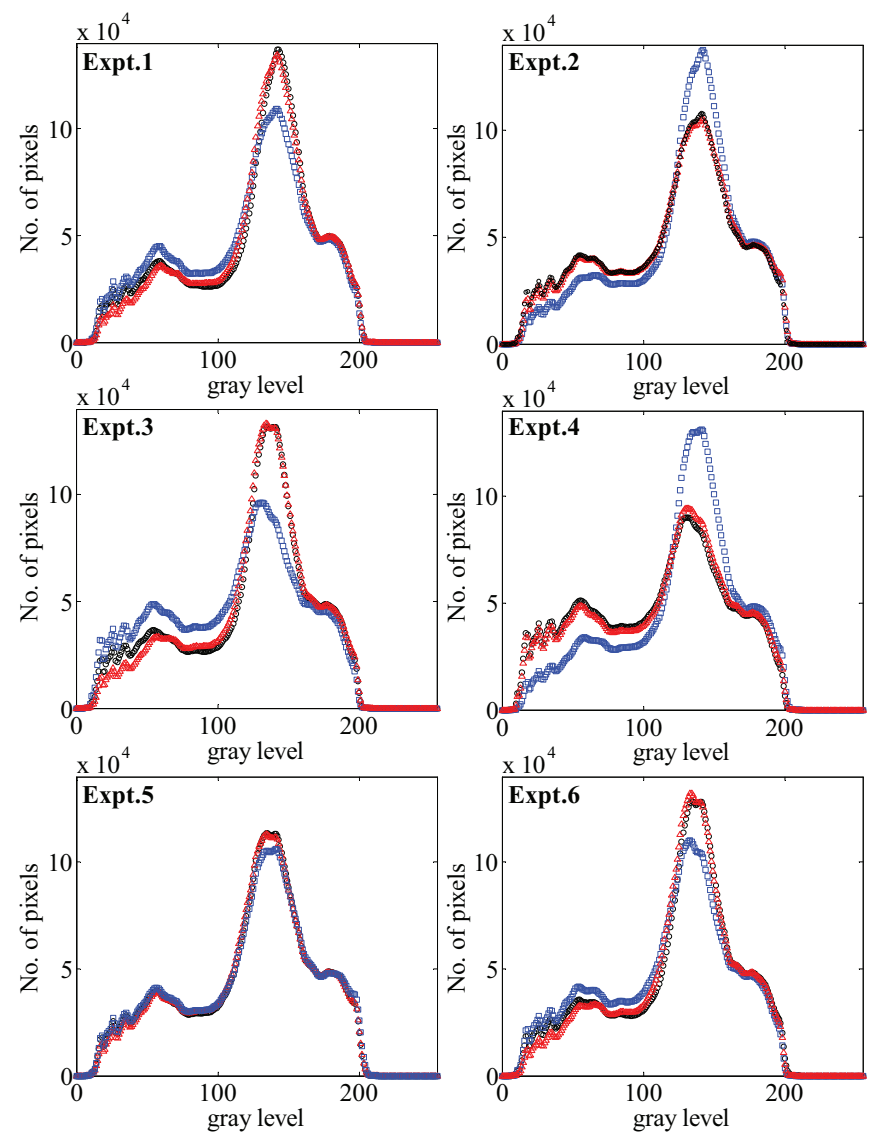

FIG. 6. (Color online) Grayscale image histograms averaged over steady-state images for the six experiments listed in Table I. Note that no image processing has been applied before obtaining these histograms. Data for steady states $\mathrm{ss}_{1}, \mathrm{ss}_{2}$, and $\mathrm{ss}_{3}$ are represented in black $(\bigcirc)$, blue $(\square)$, and red $(\triangle)$, respectively.

Image processing is performed using IMAGEJ [39]. Raw images are thresholded to obtain binary (black and white) images on which we run a standard particle analysis algorithm ${ }^{1}$ to identify air clusters and measure their sizes $n$ [40]. From steady-state images, we compute the normalized probability density functions of $n$, i.e., nonwetting cluster size distributions $\langle p(n)\rangle$, where $\langle\cdots\rangle$ represents an average over a series of $\approx 100$ images.

Figure 7 shows the distributions $\langle p(n)\rangle$ computed for the six experiments listed in Table I. These distributions typically display a power-law-like behavior with a cutoff at large cluster sizes [27,28]. As mentioned by Tallakstad et al. [27], the obtained distribution is affected by threshold values, which must thus be carefully chosen using visual inspection. Here, we focus on the variations of the distribution with the history of the system. Therefore, the most important requirement is that the image processing procedure is used consistently throughout one experiment. To avoid possible bias due to variations of illumination in the room, the experimental setup is isolated behind a dark curtain. The camera exposure time and aperture are the same for all experiments, and we use the same thresholding parameters, carefully chosen by visual

${ }^{1}$ We use the "Particles4" IMAGEJ plugin [40].
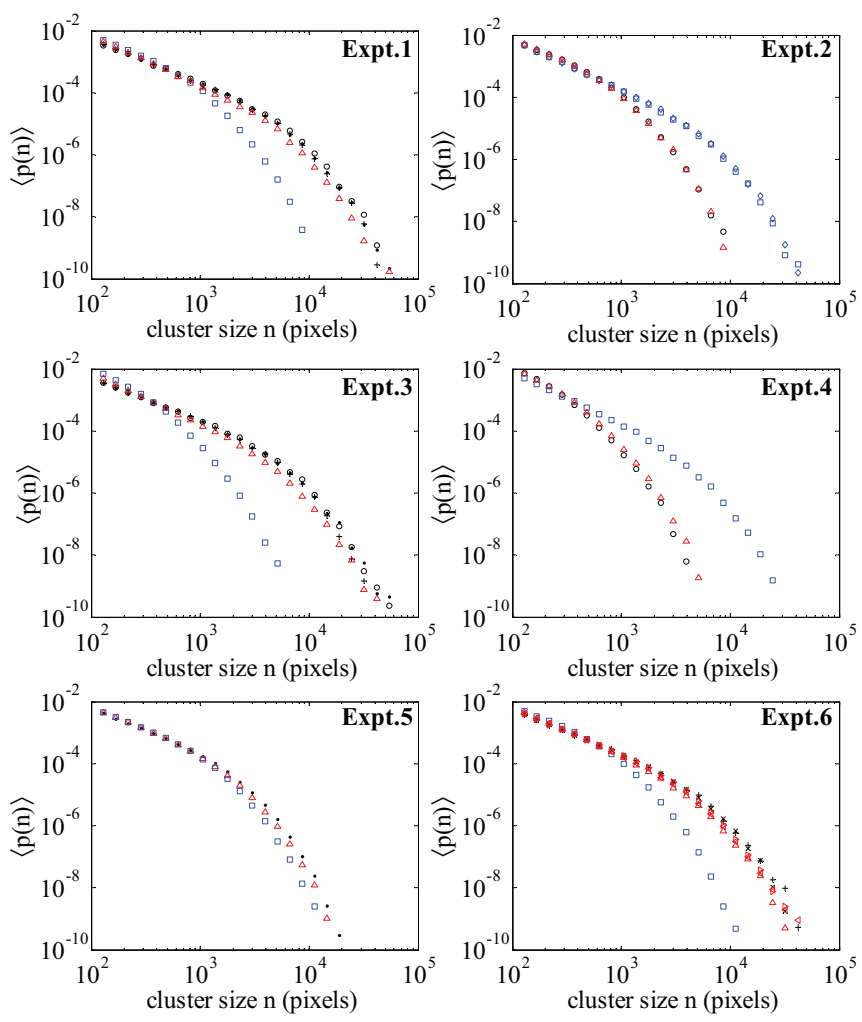

FIG. 7. (Color online) Average cluster size distributions $\langle p(n)\rangle$ computed from steady-state images. Data for steady states $\mathrm{ss}_{1}, \mathrm{ss}_{2}$, and $\mathrm{ss}_{3}$ are represented in black, blue, and red, respectively. Different symbols refer to averages performed over different series of 100 images, namely, $\bigcirc, \bigcirc$ for images in $\mathrm{ss}_{1 a},+, \times$ in $\mathrm{ss}_{1 b}, \square, \diamond$ in $\mathrm{ss}_{2} \triangle, \nabla$ in $\operatorname{ss}_{3 a}$, and $\triangleleft, \triangleright$ in $\operatorname{ss}_{3 b}$.

inspection, for all experiments. This allows us to compare images obtained in $\mathrm{ss}_{1}, \mathrm{ss}_{2}$, and $\mathrm{ss}_{3}$ for a given experiment and from one experiment to another in a meaningful way. As we observed for the histograms, it is possible to distinguish the $\mathrm{ss}_{1}$ and $\mathrm{ss}_{3}$ distributions from those corresponding to $\mathrm{ss}_{2}$ (see Fig. 7). This is coherent with the results of previous studies indicating that distributions are shifting towards higher cluster sizes when the flow rate is decreased $[27,28]$. However, the $\mathrm{ss}_{1}$ and $\mathrm{ss}_{3}$ distributions are similar, meaning that the steady-state nonwetting cluster-size distributions are history independent. We have checked that whereas varying the threshold values affects the distributions, typically by shifting them towards lower or higher cluster sizes, it does not modify the results in terms of history independence.

The experimental boundary conditions required that the controlled flow variables were the total flow rate and the fractional flows. In the next section, we turn to numerical simulations to further investigate the history dependence of the steady state for different boundary conditions, as well as higher Ca values and different viscosity ratios $M$.

\section{THE NETWORK MODEL}

The two-dimensional experimental porous medium is modeled by a network of tubes oriented at $45^{\circ}$ with respect to the overall flow direction. The tubes (or links) intersect at the vertices (or nodes) of the network with coordination 


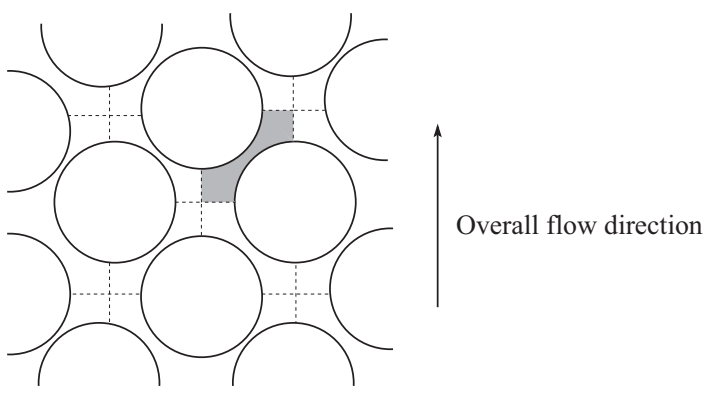

FIG. 8. Illustration of the network formed by tubes that are connected to each other through nodes where the dashed lines intersect. One single tube is colored gray. Spherical glass beads make the tubes as hourglass shaped.

number 4. The nodes are considered to have no volume, so the tubes consist of both the pore and throat volumes. The network is illustrated in Fig. 8. The disorder due to the random positions of the glass beads in the experiment is introduced in the model by choosing the radius $r$ of each tube randomly from a uniform distribution of random numbers in the range $[0.1 l, 0.4 l]$, where $l$ is the length of a tube. In order to incorporate the shape of the pores in between the spherical glass beads, each tube is considered as hourglass shaped, which introduces a capillary effect in the system. The network transports two immiscible fluids, one of which is more wetting than the other with respect to the pore walls. The fluids are separated by menisci and we obtain the capillary pressure $p_{c}$ at a meniscus inside the hourglass-shaped tubes from a modified form of the Young-Laplace law [41,42],

$$
p_{c}=\frac{2 \gamma \cos \theta}{r}\left[1-\cos \frac{2 \pi x}{l}\right],
$$

where $x$ is the position of the meniscus and $\gamma$ is the interfacial tension between the fluids. $\theta$ is the wetting angle and we consider perfectly wetting conditions, i.e., $\theta$ is either $0^{\circ}$ or $180^{\circ}$. The flow is driven by maintaining a constant total flow rate $Q$ throughout the network, which introduces a global pressure drop. The local flow rate $q$ in a tube with a pressure difference $\Delta p$ between its two ends follows the Washburn equation of capillary flow $[41,43]$

$$
q=-\frac{a k}{\mu_{\mathrm{eff}}\left(s_{\mathrm{nw}}\right) l}\left(\Delta p-\sum p_{c}\right),
$$

where $k=r^{2} / 8$ is the permeability for cylindrical tubes. Any other cross-sectional shape of the tubes will lead only to an overall geometrical factor. Here $a$ is the cross-sectional area of the tube and $\mu_{\text {eff }}\left(s_{\mathrm{nw}}\right)$ is the volume average of the viscosities of the two phases present inside the tube. Hence, it is a function of the local nonwetting saturation $s_{\mathrm{nw}}$ in that tube. The sum over $p_{c}$ runs over all the menisci inside the tube. The property that the fluid flux through every node will be zero is used to obtain the local pressures at the nodes. The set consisting of one Eq. (5) per tube, together with the Kirchhoff equations balancing the flows in and out at each node, is then solved using Cholesky factorization or the conjugate gradient method. The system is then integrated in time using an explicit Euler scheme. Inside a tube all menisci move with a speed determined by $q$. Physical processes like bubble snapoff and coalescence are introduced in the model, due to which bubbles can be formed or merged, which changes the number of menisci inside a link with time. When a meniscus reaches the end of a tube, new menisci are formed in the neighboring tubes. In each link, a total maximum number of menisci is allowed to form. When this number is exceeded, two nearest menisci are merged, keeping the volume of each fluid conserved. Here, we have considered a maximum of four menisci in one tube (i.e., two nonwetting bubbles), as it is not very likely that a lot of menisci will form in one pore, as seen from the experimental observations. Further details of the model and how the menisci are moved can be found in $[41,44]$.

In order to reach the steady state in the simulation, we considered two different approaches. The conventional way is to implement a biperiodic boundary condition (BPBC), where the links at the outlet row are connected to the inlet links, so that the network acquires a toroidal topology [26]. In this case the simulation can go forever, regardless of whether one fluid percolates or not. However, in order to keep the flow going, the global pressure gradient is maintained by considering an invisible cut through the system in terms of the pressure. Since the system is closed with this boundary condition, the individual fluid volumes remain constant throughout the simulation. The nonwetting saturation $S_{\mathrm{nw}}=V_{\mathrm{nw}} / V$ is therefore an independent parameter here, along with the total flow rate $Q$, whereas the nonwetting fractional flow $F_{\text {nw }}=Q_{\text {nw }} / Q$ fluctuates over time.

Implementing the biperiodic boundary conditions is of course impossible in experiments. As described before, in the experimental setup, two fluids are injected at one edge of the Hele-Shaw cell through a series of alternate inlets and the opposite edge is kept open. In this case, the flow rates of the two fluids can be controlled independently. Thus, the control parameters are the total flow rate $Q$ and the fractional flow $F_{\mathrm{nw}}$, whereas the saturation $S_{\mathrm{nw}}$ fluctuates. In order to have a close emulation of the experimental ensemble, we also implement open boundary conditions (OBCs) in the simulations, where the individual flow rates at the inlet links are controlled. Therefore, with OBCs, the system is open in the direction of total flow while we consider periodic boundary conditions in the direction perpendicular to the overall flow.

\section{SIMULATION RESULTS}

Simulations are performed considering networks of $256 \times$ 256 links for BPBCs and $128 \times 192$ links for OBCs. In order to avoid any traces from the inlets with OBCs, only a $128 \times 128$ segment of the network towards the outlets is considered for the analysis (see Fig. 10). Each link has a length of $1 \mathrm{~mm}$, which is equal to the bead diameter used in the experiments. Most of the simulations are performed for the viscosity ratio $M=$ 1. Three different capillary numbers, $\mathrm{Ca}_{1}=1.92 \times 10^{-5}$, $\mathrm{Ca}_{2}=9.15 \times 10^{-3}$, and $\mathrm{Ca}_{3}=2.88 \times 10^{-2}$ are considered for BPBCs. For OBCs, the capillary numbers considered are $\mathrm{Ca}_{1}=3.2 \times 10^{-3}, \mathrm{Ca}_{2}=3.2 \times 10^{-2}$ and $\mathrm{Ca}_{3}=7.0 \times 10^{-2}$. For OBCs we choose a similar fractional flow $F_{\text {nw }}=0.5$ as in the experiments. With BPBCs, we run the simulation for the saturation $S_{\mathrm{nw}}=0.74$ which we find close to the critical point for the range of parameters we considered here [32]. We will report only one set of simulations for $M=10^{-4}$ with BPBCs 
for a system with $128 \times 128$ links and $S_{\text {nw }}=0.5$ with capillary numbers $5.06 \times 10^{-2}$ and $1.24 \times 10^{-1}$, as the conjugate gradient solver converges very slowly for viscosity-unmatched fluids, making the simulation very computationally expensive.

To investigate the history dependence, we use a procedure analogous to the experimental one (see Sec. III A): The simulation is started from the initial condition with a capillary number setting a constant flow rate $Q_{1}$. Once a steady state $\mathrm{ss}_{1}$ is reached, the capillary number is altered to a different value setting another constant flow rate $Q_{2}$, and this new flow rate is maintained until a different steady state $\mathrm{ss}_{2}$ is reached. Finally, the capillary number is set back to the initial value and the system is allowed to evolve towards a third steady state $\mathrm{ss}_{3}$. Each simulation thus involves two different capillary numbers among $\mathrm{Ca}_{1}, \mathrm{Ca}_{2}$, and $\mathrm{Ca}_{3}$, and therefore six different simulations have been performed for each boundary condition. The steady states are compared using three different criteria - the average pressure drop, the distribution of fluid saturation over the system, and the nonwetting cluster-size distribution. All the measurements are averaged over 50 to 100 configurations in the steady state and 5 to 10 different realizations of the network.

Fluid morphologies for a typical simulation in the three steady states $\mathrm{ss}_{1}, \mathrm{ss}_{2}$, and $\mathrm{ss}_{3}$ are shown on Figs. 9 and 10 for BPBCs and OBCs, respectively. Here the simulation starts from $\mathrm{Ca}=9.15 \times 10^{-3}$ to reach $\mathrm{ss}_{1}$, then it is altered to $\mathrm{Ca}=2.88 \times 10^{-2}$ to reach $\mathrm{ss}_{2}$, and then it is again turned back to the initial $\mathrm{Ca}=9.15 \times 10^{-3}$ to reach $\mathrm{ss}_{3}$. Figures 9 and 10 show the distributions of saturation over the network in these three steady states in panels (a), (b), and (c) respectively, where the grayscales from black to white correspond to $s_{\mathrm{nw}}=1$ to 0 inside a link. For BPBCs, it is not possible to see any difference in the grayscale saturation distributions, as the system is closed
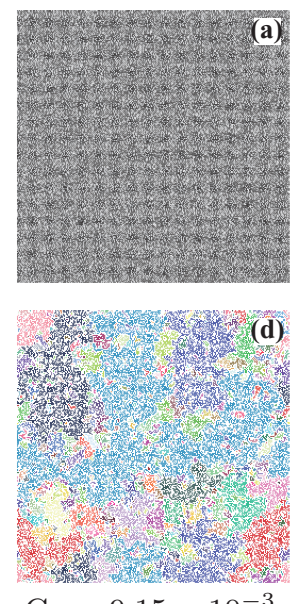

$\mathrm{Ca}=9.15 \times 10^{-3}$
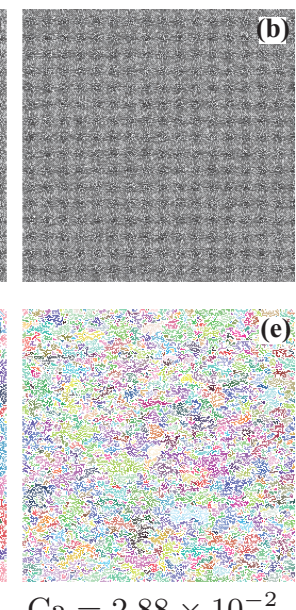

$\mathrm{Ca}=2.88 \times 10^{-2}$
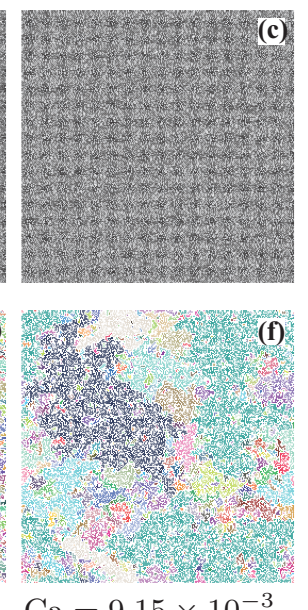

FIG. 9. (Color online) Typical steady-state fluid morphology over the network with BPBCs for $M=1$ and $S_{\text {nw }}=0.74$. The distribution of nonwetting fluid saturation inside the links in the steady states $\mathrm{ss}_{1}$, $\mathrm{ss}_{2}$, and $\mathrm{ss}_{3}$ are illustrated in (a), (b), and (c), respectively, and the corresponding capillary numbers are indicated under each column. The grayscales from black to white correspond to the non-wetting saturation from 1 to 0 inside a link. The nonwetting clusters identified by the Hoshen-Kopelman algorithm are shown by different random colors in (d), (e), and (f).
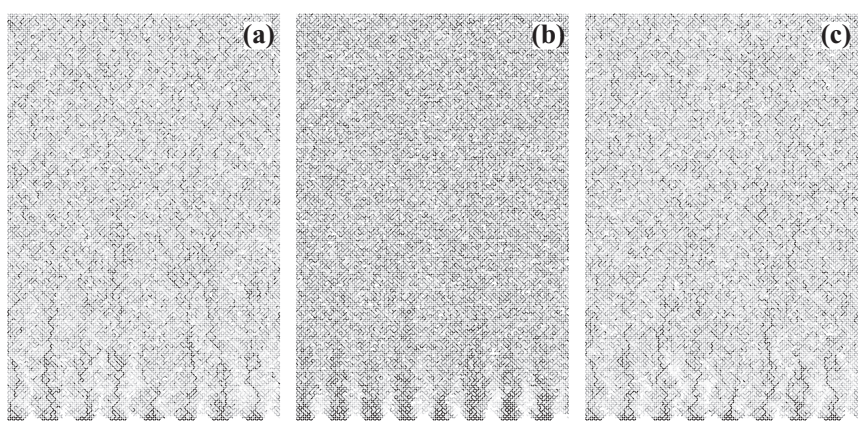

(d)

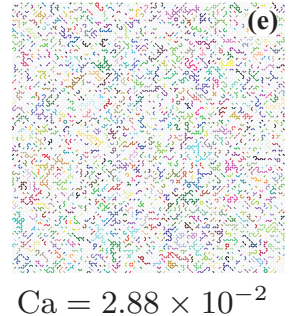

e)

(f)

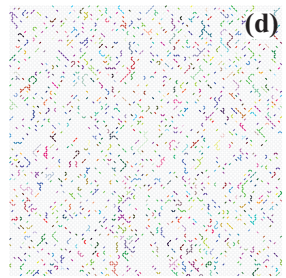

$\mathrm{Ca}=9.15 \times 10^{-3}$

$\mathrm{Ca}=2.88 \times 10^{-2}$

$\mathrm{Ca}=9.15 \times 10^{-3}$

FIG. 10. (Color online) Steady-state fluid morphology over the network in OBCs for $M=1$ and $F_{\text {nw }}=0.5$. The figures are drawn in the similar way as for BPBCs.

and the total saturation is conserved. However, as we will see, identifying the clusters allows us to distinguish $\mathrm{ss}_{1}$ and $\mathrm{ss}_{3}$ from $\mathrm{ss}_{2}$ [see Figs. 9(d)-9(f)]. In OBCs, the saturation distributions look similar in $\mathrm{ss}_{1}$ and $\mathrm{ss}_{3}$ whereas $\mathrm{ss}_{2}$ shows more nonwetting saturation than the others. This is consistent with previous numerical studies showing that the variation of saturation with $\mathrm{Ca}$ depends on the viscosity ratio, fractional flow, and other flow parameters [26]. We then identify the nonwetting clusters using the Hoshen-Kopelman algorithm [45]. As every link can be occupied by both the fluids, a clip threshold in the link saturation is considered to identify the clusters [46]. If a neighboring link has a nonwetting saturation higher than the clip threshold, the link is then considered to belong to the same cluster. The clusters are shown in panels (d), (e) and (f) of Figs. 9 and 10 for the three steady states, where each cluster is drawn in a different color, chosen randomly. The distribution of the clusters shows a clear characteristic difference of $\mathrm{ss}_{1}$ and $\mathrm{ss}_{3}$ from $\mathrm{ss}_{2}$ in both $\mathrm{BPBCs}$ and OBCs. The cluster sizes in $\mathrm{ss}_{1}$ and $\mathrm{ss}_{3}$ look very similar, and they are distinctly different from that of $\mathrm{ss}_{2}$.

The temporal evolution of pressure drops for different simulations is shown in Figs. $11(M=1)$ and $12\left(M=10^{-4}\right)$ for BPBCs and in Fig. 13 for OBCs. The two different capillary numbers for each simulation corresponding to $\mathrm{ss}_{1}, \mathrm{ss}_{2}$, and $\mathrm{ss}_{3}$ are indicated in the plots. With BPBCs, we measure the global pressure drop $\Delta P$ over the whole system. With OBCs, we measure the pressure drops $\Delta P_{i}$ at the inlet nodes and $\Delta P_{m}$ at the middle of the system, with respect to the outlet where the pressures are averaged over all the nodes in the corresponding row, in the direction perpendicular to the flow. We observe very similar behaviors in the pressure curves as in the experiments. In the case of BPBCs, the system is initialized by filling the tubes with the fluids randomly at the desired saturation $S_{\mathrm{nw}}$, which will be constant throughout the simulation. This random initialization has the advantage of decreasing the simulation time significantly, since the steady state is reached faster than 

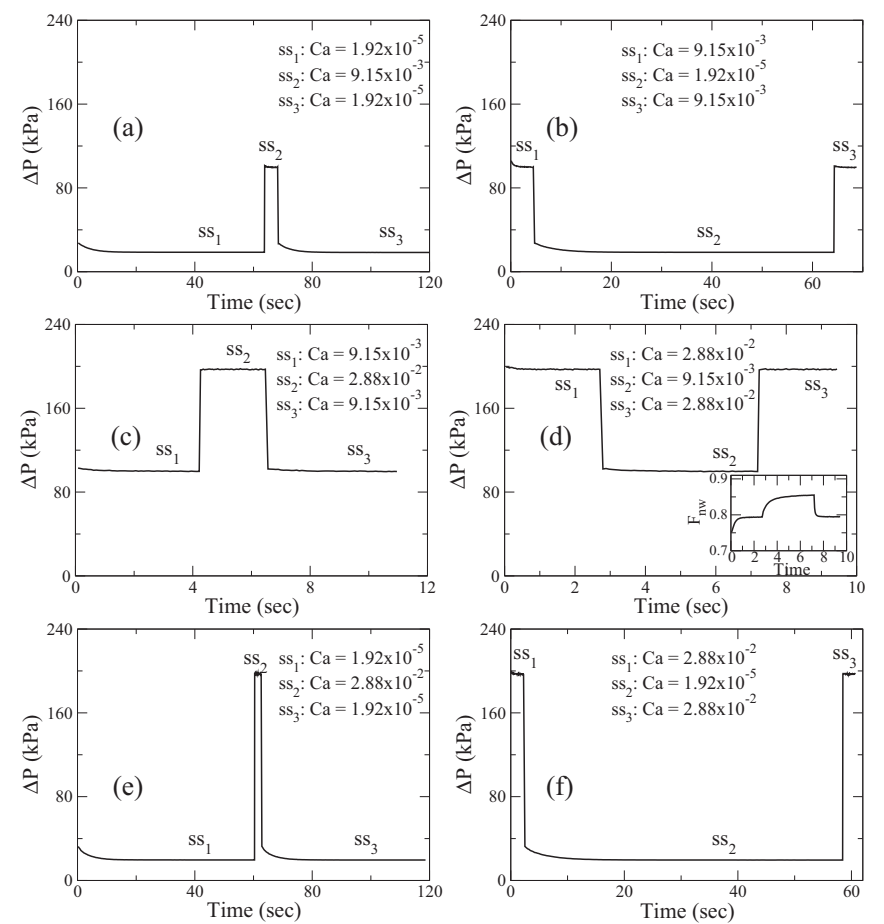

FIG. 11. Global pressure drop $\Delta P$ as a function of time for BPBCs in different simulations with $M=1$ and $S_{\mathrm{nw}}=0.74$. A rapid change in $\Delta P$ can be observed as soon as the overall flow rate is altered. When the flow rate is restored to the initial value, $\Delta P$ settles back to the initial steady-state value as seen for $\mathrm{ss}_{1}$ and $\mathrm{ss}_{3}$. Other global parameters like the nonwetting fractional flow $F_{\text {nw }}$ also settle back to the initial steady-state value as shown in the inset of (d).

with an initial condition in which the two fluids are completely segregated. Due to this initial random filling in BPBCs, the global pressure $\Delta P$ starts from a higher value and decreases with time due to the formation of clusters. Subsequently, it reaches the steady state $\mathrm{ss}_{1}$ and $\Delta P$ fluctuates around a constant average value as seen on Figs. 11 and 12. With OBCs, the system is initialized by saturating the network completely with the wetting fluid, and then the simulation is started by injecting two fluids simultaneously through a series of alternate inlets. The flow rates of individual fluids are controlled to obtain the required fractional flow $F_{\mathrm{nw}}$. Both drainage and imbibition therefore take place at the pore level, creating new menisci, which increase the pressure drop with time as seen in Fig. 13. Away from the inlets, the trace of the injection channels vanishes, and a steady-state $\mathrm{ss}_{1}$ is attained, and $\Delta P_{i}$ and $\Delta P_{m}$ fluctuate around constant average values. Next, as soon as
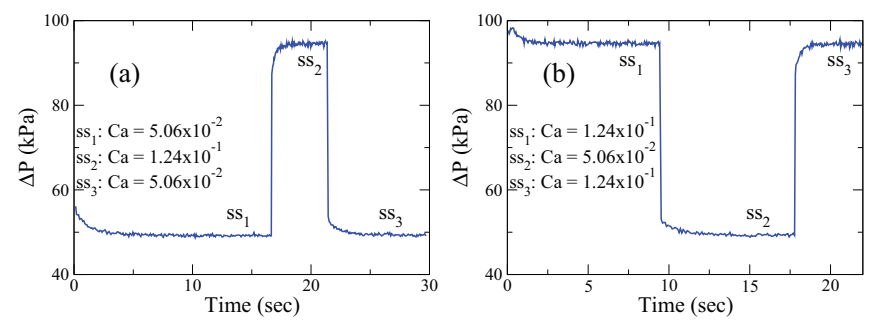

FIG. 12. (Color online) Global pressure drop $\Delta P$ with time for BPBCs with $M=10^{-4}$ and $S_{\text {nw }}=0.5$.
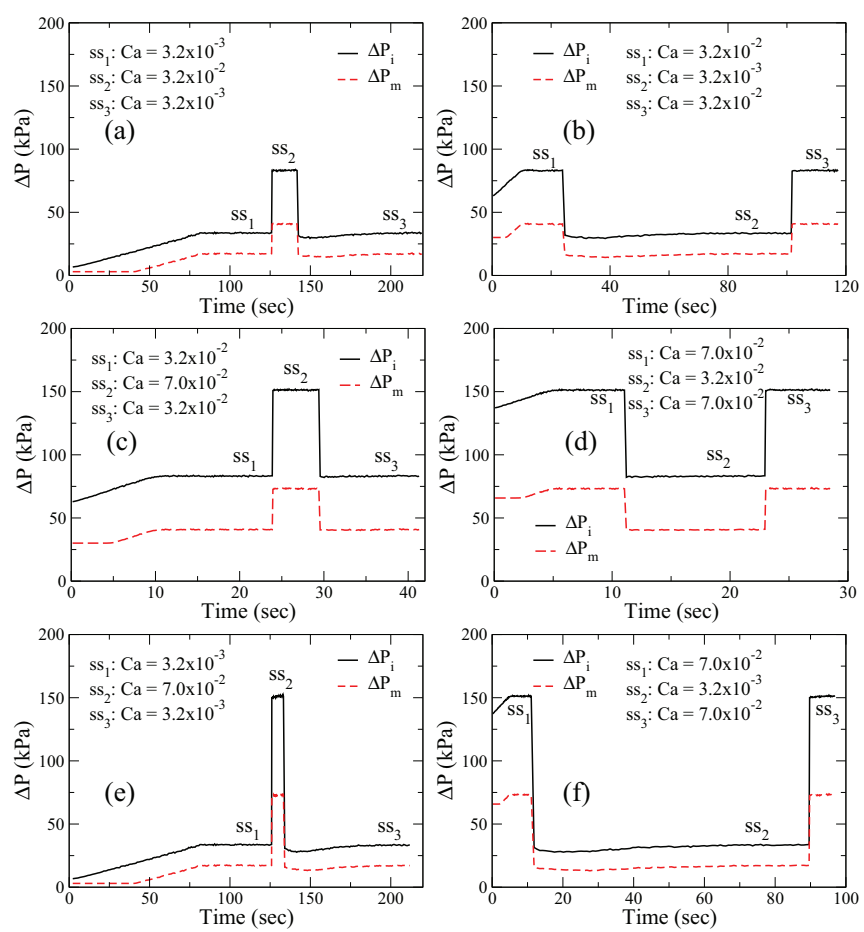

FIG. 13. (Color online) Time evolution of global pressure drops $\Delta P_{i}$ [black (upper) line] at the inlet and $\Delta P_{m}$ [red (lower) line] at the middle of the system for OBCs with $M=1$. A rapid change in both the pressure drops can be observed as soon as $\mathrm{Ca}$ is altered, but they again settle back to the initial value when the flow rate is restored to the initial one.

the capillary number is changed to a different value, a rapid change in the pressure drops is observed for both BPBCs and $\mathrm{OBCs}$, and the new steady state $\mathrm{ss}_{2}$ is reached, characterized by different constant values in the average pressure drops. When the capillary number is again altered to the initial value to reach the steady state $\mathrm{ss}_{3}$, we find that the global pressure drops change back to the initial average value. Moreover, the global pressure drops corresponding to the same capillary numbers in different simulations have the same average value, no matter from which conditions they have been reached. Other global flow properties, like the nonwetting fractional flow $F_{\text {nw }}$ with BPBCs, also change to another steady-state value as $\mathrm{Ca}$ is altered, but again return back to the same initial value as soon as $\mathrm{Ca}$ is turned back to the initial value. This is shown in the inset of Fig. 11(d).

The global pressure estimates therefore completely support the experimental observations, i.e., that the steady state depends only on the imposed flow rate and not on the initial condition. Now, in order to find detailed microscopic information in this regard, we measure the distribution of link saturation over the system. This measurement provides us with similar information to that from experimental grayscale image histograms, despite being computed slighly differently. More precisely, in the experiment, the grayscale of each pixel is counted, where one pixel corresponds to any one of the three components - the viscous fluid, air, or the glass beads. In the simulation, on the other hand, we count the nonwetting saturation inside each link and compute the histogram of the link counts. Therefore, one should not try to make a direct 

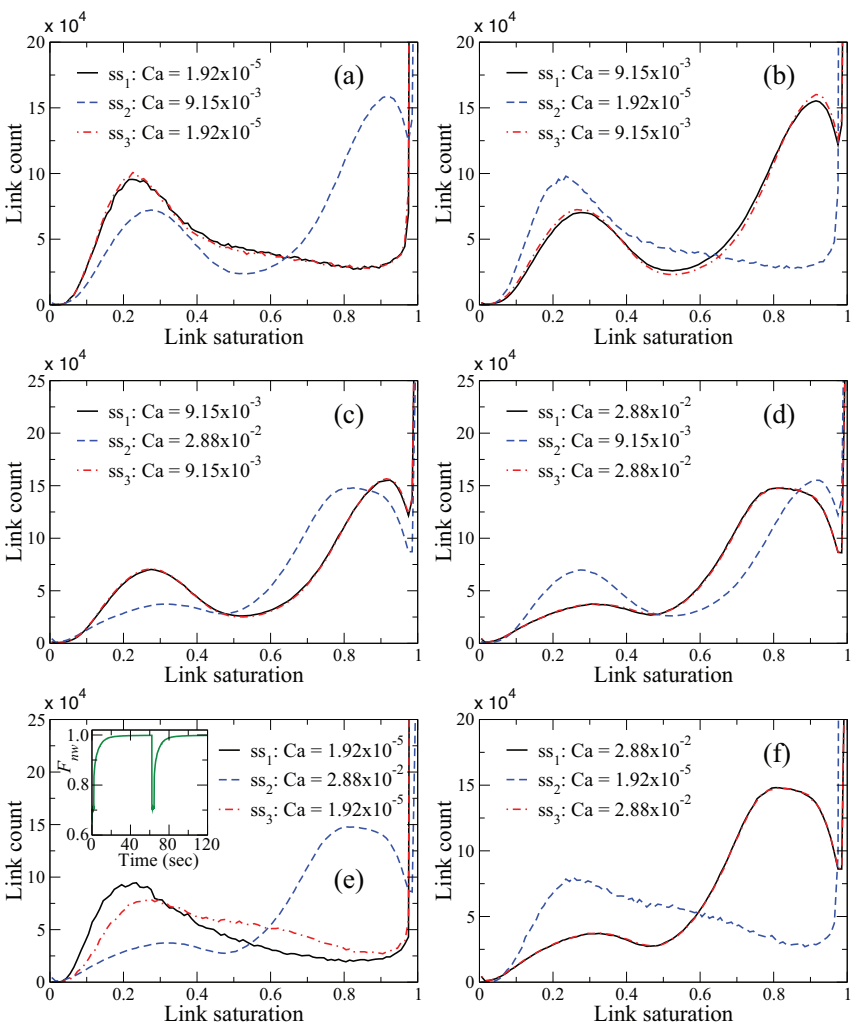

FIG. 14. (Color online) Histograms of the network links according to nonwetting fluid saturation inside the links over the steady-state configurations for BPBCs with $M=1$ and $S_{\mathrm{nw}}=0.74$. In all the simulations, the histograms are found to be similar for $\mathrm{ss}_{1}$ [black (solid) line] and $\mathrm{ss}_{3}$ [red (dash-dotted) line]. Two distinct peaks are observed for $\mathrm{Ca}=9.15 \times 10^{-3}$, implying that the links are highly saturated with either the nonwetting or the wetting fluid. The inset of (e) shows the nonwetting fractional flow $F_{\text {nw }}$ for the corresponding simulation where $F_{\mathrm{nw}} \approx 0.99$ at $\mathrm{Ca}=1.92 \times 10^{-5}$, which is the reason for the minor variation in the histogram patterns for $\mathrm{ss}_{1}$ and $\mathrm{ss}_{3}$.

match of the histogram patterns from the experiments to the simulations. The histograms in the three steady states $\mathrm{ss}_{1}$, $\mathrm{ss}_{2}$, and $\mathrm{ss}_{3}$ in different simulations are plotted on Figs. 14 $(M=1)$ and $15\left(M=10^{-4}\right)$ for BPBCs and 16 for OBCs. In each simulation, it is very clear that the histograms for $\mathrm{ss}_{1}$ and $\mathrm{ss}_{3}$ fall on each other, whereas they are distinctly different from that of $\mathrm{ss}_{2}$. Moreover, the histogram patterns corresponding to the same $\mathrm{Ca}$ in different simulations are identical. A minor difference in the histograms for $\mathrm{ss}_{1}$ and $\mathrm{ss}_{3}$ is observed only for $\mathrm{Ca}=1.92 \times 10^{-5}$ with BPBCs. This
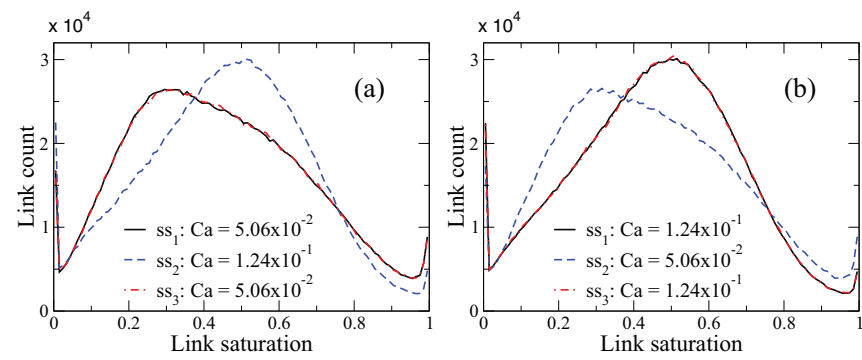

FIG. 15. (Color online) Steady-state saturation histograms for BPBCs with $M=10^{-4}$ and $S_{\mathrm{nw}}=0.5$.
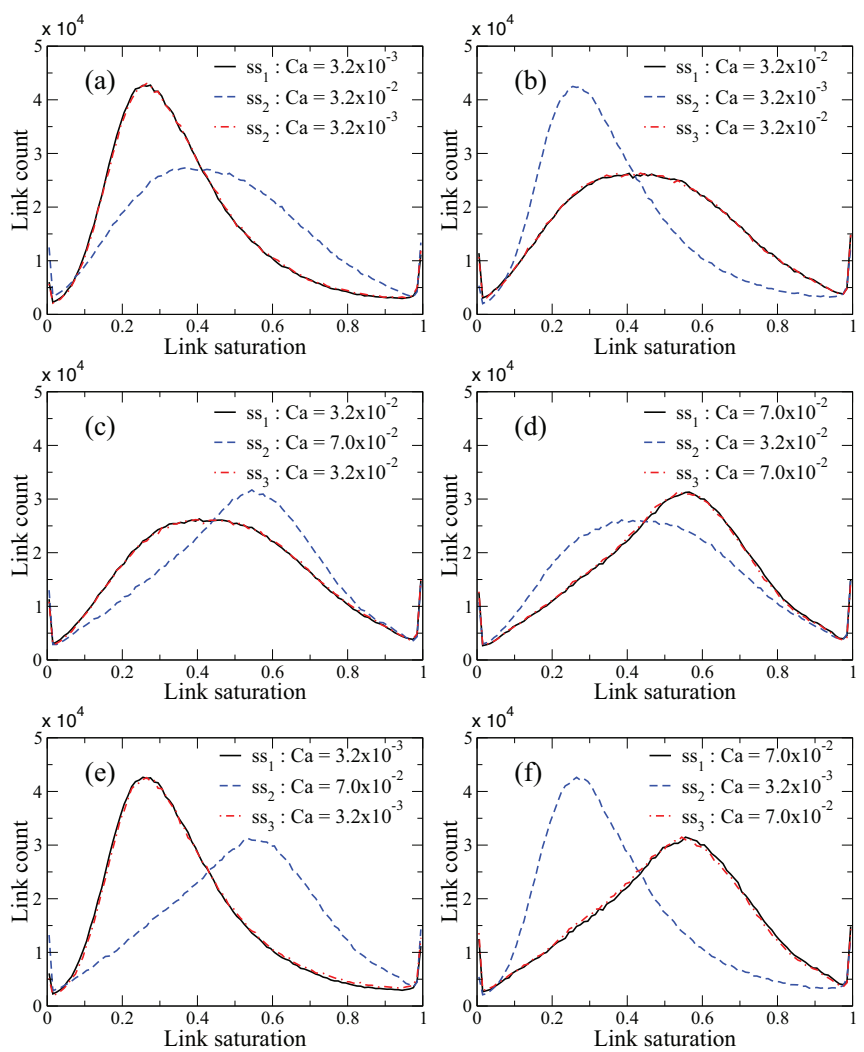

FIG. 16. (Color online) Histograms of the links according to the nonwetting fluid saturation inside the links over the steady-state configurations for $\mathrm{OBCs}$ with $M=1$. In all the simulations, the histograms are found to be identical for $\mathrm{ss}_{1}$ [black (solid) line] and $\mathrm{ss}_{3}$ [red (dash-dotted) line]. The histograms contain only one peak around the middle, which means that the links are mostly saturated with a mixture of both the fluids.

is due to the appearance, at low $\mathrm{Ca}$ and high saturation with BPBCs, of percolating nonwetting flow channels yielding a high nonwetting fractional flow $\left(F_{\mathrm{nw}} \approx 0.99\right)$, as shown in the inset of Fig. 14(e), while the rest of the system is immobilized.

On the histograms, we observe two distinct peaks with BPBCs for $\mathrm{Ca}=9.15 \times 10^{-3}$ : one at $s_{\text {nw }} \gtrsim 0.8$ corresponding to the links mostly filled with nonwetting fluid, and the other at $s_{\text {nw }} \lesssim 0.4$ corresponding to the links mostly filled with wetting fluid. Therefore links can be divided into two categories: highly saturated with either the nonwetting or the wetting fluid, rather than containing a mixture of the two phases. This in turn indicates the presence of large clusters at this $\mathrm{Ca}$, as already observed in the fluid morphology on Figs. 9(d) and 9(f). For the other two Ca values with BPBCs, the histograms are changing towards having one peak, with a flatter shape. For $M=10^{-4}$ in BPBCs, and in OBCs, the histograms also display one peak, roughly centered but whose position shifts along the $x$ axis with changing $\mathrm{Ca}$. This indicates that most of the links are filled with a mixture of both fluids. Therefore it is difficult to obtain large clusters in such conditions, as observed in the fluid morphology for OBCs (Fig. 10).

Finally we compute the nonwetting cluster-size distributions at different steady states. Here, the size $n$ of a cluster is defined by the total number of links that belong to the cluster. The probability $p(n)$ to have an $n$-sized cluster is then 

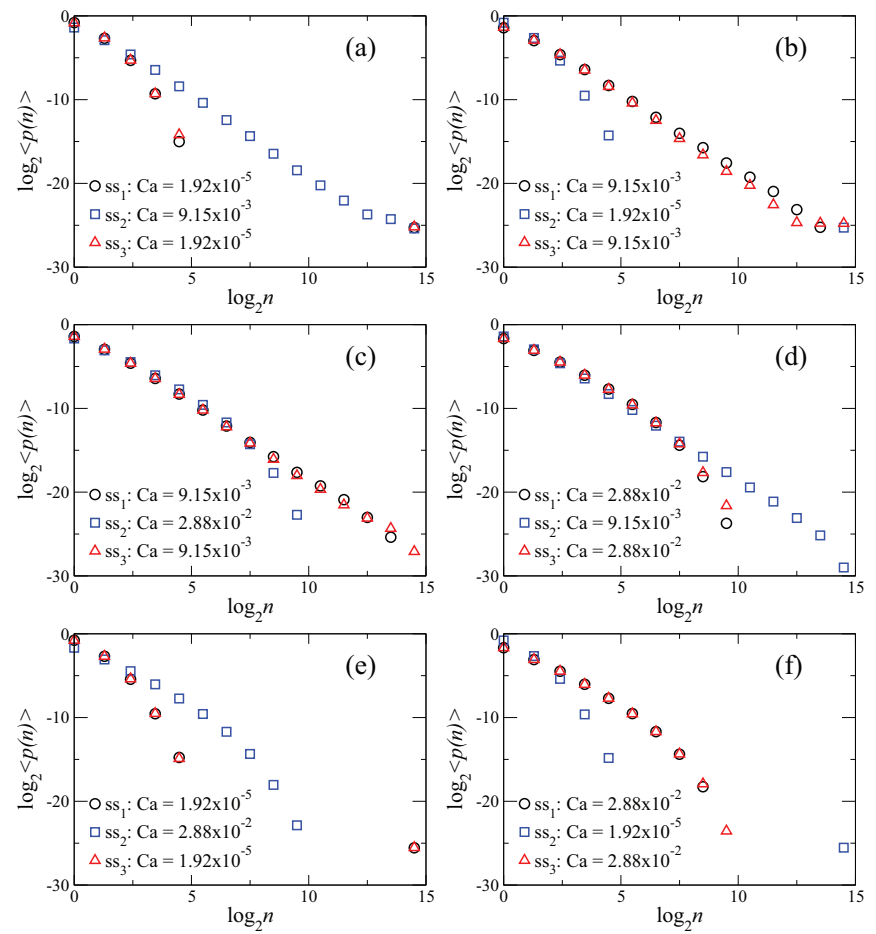

FIG. 17. (Color online) Steady-state nonwetting cluster-size distributions $\langle p(n)\rangle$ vs $n$ for BPBCs with $M=1$ and $S_{\mathrm{nw}}=0.74$. For all the simulations, $\mathrm{ss}_{1}$ and $\mathrm{ss}_{3}$ are found to have the same distributions, which depend only on the capillary number.

defined as $p(n)=N(n) / N_{\text {tot }}$, where $N(n)$ is the number of $n$-sized clusters out of a total of $N_{\text {tot }}$ clusters identified. $p(n)$ is averaged over different configurations in the steady state and different samples of the network. In Figs. 17 and 18, $\langle p(n)\rangle$ is plotted on a log-log scale for BPBCs and in Fig. 19 for OBCs, for different simulations. For all the simulations, we find that the cluster-size distributions are identical for $\mathrm{ss}_{1}$ and $\mathrm{ss}_{3}$, whereas they are different for $\mathrm{ss}_{2}$. Distributions for the same $\mathrm{Ca}$ for different simulations are also the same, showing that the steady-state cluster-size distributions are history independent.

\section{CONCLUSIONS}

In this article we have considered the question of history dependence in steady-state two-phase flow in porous media and presented detailed experimental and numerical investigations in this context. Experimentally, a quasi-two-dimensional laboratory model consisting of a Hele-Shaw cell filled with glass beads is considered, through which two phases, a
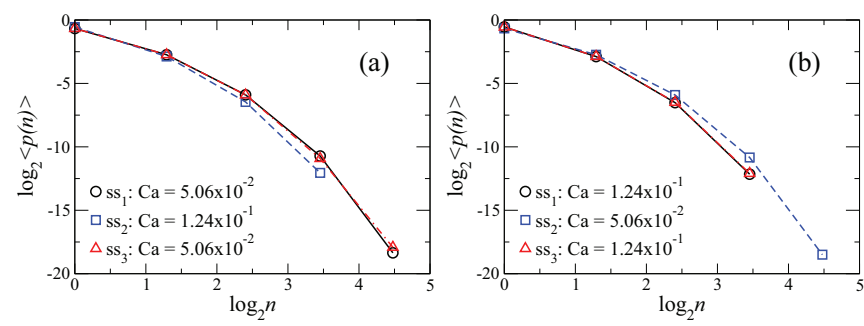

FIG. 18. (Color online) Steady-state nonwetting cluster-size distributions $\langle p(n)\rangle$ vs $n$ for BPBCs with $M=10^{-4}$ and $S_{\text {nw }}=0.5$.
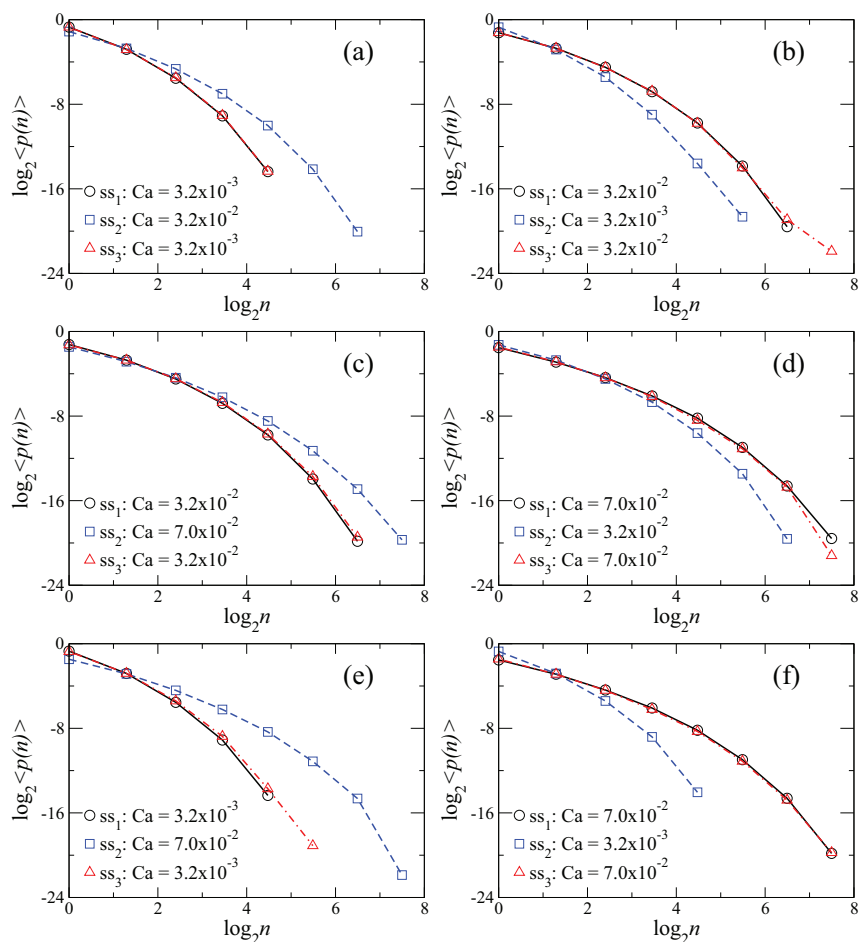

FIG. 19. (Color online) Steady-state nonwetting cluster-size distributions $\langle p(n)\rangle$ vs $n$ for OBCs with $M=1$. As for BPBCs, the distributions are found to be identical in $\mathrm{ss}_{1}$ and $\mathrm{ss}_{3}$ in all the simulations.

gas-liquid pair with a viscosity ratio of $10^{-4}$ flows at a constant flow rate. The system is allowed to evolve to a steady state where the global pressure drop fluctuates around a constant average value. Steady states corresponding to the same control parameters (e.g., capillary number) are attained from different initial conditions. In order to characterize the complex flow patterns in the steady state, grayscale histograms of snapshots and the nonwetting cluster-size distributions are analyzed. The system is then modeled numerically by a network of disordered pores transporting two immiscible fluids. The steady-state situation in the network model is attained by implementing two different boundary conditions, a toroidal one with constant saturation and an open boundary with constant fractional flow, as in the experiments. The global pressure drop, distribution of nonwetting pore saturation over the network, and cluster-size distributions are computed. Both the experimental and numerical results show that when both the fluids are flowing in steady state, different measurements corresponding to the same control parameters are identical, no matter how the steady state has been reached. Thus, unlike the transients, the steady states depend only on the external parameters, but do not depend on the initial preparation of the system or the history of the process. We therefore conclude that, within the range of parameters explored in this study, the steady states in simultaneous flow of two phases through a porous medium are history independent.

However, the present study covers only a restricted range of the parameter space. To obtain a complete picture of steady state in simultaneous two-phase flow in 2D porous media, additional work is needed. First, in the present work, 
we have used only systems with a relatively high porosity. Second, the use of a gas-liquid system with a high viscosity contrast $\left(M \approx 10^{-4}\right)$ yields a specific dynamical steady-state regime in which flow patterns vary in time. In future work, it will be very interesting to investigate in detail how both the compressibility of the gas and the high viscosity contrast force the system to explore different configurations. In terms of a thermodynamic description, in particular, this is potentially making the system ergodic. Using an experimental porous medium similar to the one presented here, we are currently running experiments with two liquids, thereby investigating the situation $M \approx 1$. Preliminary results seem to show that film flow is an important contribution to the steady-state dynamics in this case. Although the simulations presented here investigate the case $M=1$, they are performed by neglecting film flow. Thus, the implementation of film flow in the numerical model will be of great interest in the future. Finally, it is by no means obvious how to extend these results to $3 \mathrm{D}$ cases, and the development of 3D experiments in model porous media is important for future work.

\section{ACKNOWLEDGMENTS}

This work was supported by the Research Council of Norway (NFR) through PETROMAKS Project No. 193298 and CLIMIT Project No. 200041. We thank Signe Kjelstrup, Dick Bedeaux, Mihailo Jankov, and Olav Aursjø for fruitful discussions.
[1] D. G. Avraam and A. C. Payatakes, J. Fluid Mech. 293, 207 (1995).

[2] J. Bonnet and R. Lenormand, Rev. Inst. Fr. Pet. 32, 477 (1977).

[3] K. J. Måløy, J. Feder, and T. Jøssang, Phys. Rev. Lett. 55, 2688 (1985).

[4] O. I. Frette, K. J. Måløy, J. Schmittbuhl, and A. Hansen, Phys. Rev. E 55, 2969 (1997).

[5] V. Berejnov, N. Djilali, and D. Sinton, Lab Chip 8, 689 (2008).

[6] J. Koplik and T. J. Lasseter, Soc. Pet. Eng. J. 25, 89 (1985).

[7] R. Lenormand, E. Touboul, and C. Zarcone, J. Fluid Mech. 189, 165 (1988).

[8] M. Blunt and P. King, Phys. Rev. A 42, 4780 (1990).

[9] G. N. Constantinides and A. C. Payatakes, J. Colloid Interface Sci. 141, 486 (1991).

[10] G. N. Constantinides and A. C. Payatakes, AIChE J. 42, 369 (1996).

[11] D. H. Rothman, J. Geophys. Res. 95, 8663 (1990).

[12] A. K. Gunstensen, D. H. Rothman, S. Zaleski, and G. Zanetti, Phys. Rev. A 43, 4320 (1991).

[13] A. K. Gunstensen and D. H. Rothman, J. Geophys. Res. 98, 6431 (1993).

[14] B. Ferréol and D. H. Rothman, Transp. Porous Media 20, 3 (1995).

[15] H. Liu, A. J. Valocchi, and Q. Kang, Phys. Rev. E 85, 046309 (2012).

[16] O. Aursjø, H. A. Knudsen, E. G. Flekkøy, and K. J. Måløy, Phys. Rev. E 82, 026305 (2010).

[17] O. Aursjø, G. Løvoll, H. A. Knudsen, E. G. Flekkøy, and K. J. Måløy, Transp. Porous Media 86, 125 (2011).

[18] D. Wilkinson and J. F. Willemsen, J. Phys. A 16, 3365 (1983).

[19] T. A. Witten and L. M. Sander, Phys. Rev. Lett. 47, 1400 (1981).

[20] L. Paterson, Phys. Rev. Lett. 52, 1621 (1984).

[21] P. Binning and M. A. Celia, Adv. Water Resour. 22, 461 (1999).

[22] D. G. Avraam and A. C. Payatakes, Transp. Porous Media 20, 135 (1995).

[23] D. G. Avraam and A. C. Payatakes, Ind. Eng. Chem. Res. 38, 778 (1999).

[24] C. D. Tsakiroglou, D. G. Avraam, and A. C. Payatakes, Adv. Water Resour. 30, 1981 (2007).
[25] M. S. Valavanides, G. N. Constantinides, and A. C. Payatakes, Transp. Porous Media 30, 267 (1998).

[26] H. A. Knudsen and A. Hansen, Phys. Rev. E 65, 056310 (2002).

[27] K. T. Tallakstad, G. Løvoll, H. A. Knudsen, T. Ramstad, E. G. Flekkøy, and K. J. Måløy, Phys. Rev. E 80, 036308 (2009).

[28] K. T. Tallakstad, H. A. Knudsen, T. Ramstad, G. Løvoll, K. J. Måløy, R. Toussaint, and E. G. Flekkøy, Phys. Rev. Lett. 102, 074502 (2009).

[29] E. M. Rassi, S. L. Codd, and J. D. Seymour, New J. Phys. 13, 015007 (2011).

[30] S. Sinha, A. Hansen, D. Bedeaux, and S. Kjelstrup, Phys. Rev. E 87, 025001 (2013).

[31] S. Sinha and A. Hansen, Europhys. Lett. 99, 44004 (2012).

[32] T. Ramstad and A. Hansen, Phys. Rev. E 73, 026306 (2006).

[33] A. Hansen and T. Ramstad, Comput. Geosci. 13, 227 (2009).

[34] R. Juanes, E. J. Spiteri, F. M. Orr, Jr., and M. J. Blunt, Water Resour. Res. 42, W12418 (2006).

[35] S. A. Aryana and A. R. Kovscek, Phys. Rev. E 86, 066310 (2012).

[36] T. J. Greytak, R. T. Johnson, D. N. Paulson, and J. C. Wheatley, Phys. Rev. Lett. 31, 452 (1973).

[37] J. Watson and D. S. Fisher, Phys. Rev. B 54, 938 (1996).

[38] N.-S. Cheng, Ind. Eng. Chem. Res. 47, 3285 (2008).

[39] W. S. Rasband, computer code IMAGEJ (U.S. National Institutes of Health, Bethesda, MD, USA, 1997-2012), http://imagej.nih.gov/ij/.

[40] G. Landini, http://www.dentistry.bham.ac.uk/landinig.

[41] E. Aker, K. J. Måløy, A. Hansen, and G. G. Batrouni, Transp. Porous Media 32, 163 (1998); E. Aker, K. J. Måløy, and A. Hansen, Phys. Rev. E 58, 2217 (1998).

[42] F. A. L. Dullien, Porous Media: Fluid Transport and Pore Structure (Academic Press, San Diego, 1992).

[43] E. W. Washburn, Phys. Rev. 17, 273 (1921).

[44] H. A. Knudsen, E. Aker, and A. Hansen, Transp. Porous Media 47, 99 (2002).

[45] J. Hoshen and R. Kopelman, Phys. Rev. B 14, 3438 (1976).

[46] G. Tørå, T. Ramstad, and A. Hansen, Europhys. Lett. 87, 54002 (2009). 